\title{
Adjunctive therapy for the treatment of primary generalized tonic-clonic seizures: focus on once- daily lamotrigine
}

This article was published in the following Dove Press journal:

Drug Design, Development and Therapy

17 November 2010

Number of times this article has been viewed

\author{
Linda Steinbaugh \\ Jerzy P Szaflarski \\ Department of Neurology and \\ Cincinnati Epilepsy Center, University \\ of Cincinnati Academic Health Center, \\ Cincinnati, Ohio, USA
}

\begin{abstract}
Idiopathic generalized epilepsies are frequently encountered by neurologists, and providing an accurate diagnosis and effective treatment(s) are the necessary components of successful patient care. With the introduction of new antiepileptic medications, physicians are better equipped for this goal. The immediate-release formulation of lamotrigine (LTG-IR) has been approved for primary generalized tonic-clonic seizures since 2006. The extended-release formulation of lamotrigine (LTG-XR) was approved for adjunctive therapy in patients with primary generalized tonic-clonic seizures in 2010. Although its exact mechanism of action is not yet fully elucidated, studies have demonstrated multiple possible pathways. Although both the LTG-IR and LTG-XR formulations have similar side effects and are generally well tolerated, LTG-XR may be preferable for its ease of use, which may increase patient compliance and decrease fluctuations in serum drug levels. The ease of conversion between the formulations also makes lamotrigine an attractive treatment option for patients with primary generalized tonicclonic seizures. LTG-IR has demonstrated efficacy in treatment-resistant idiopathic generalized epilepsies in both adults and children. Although there are still some questions regarding all possible applications of LTG-XR, as further research is being done, it is clear that LTG-XR may hold some advantages when compared with other anticonvulsants.
\end{abstract}

Keywords: lamotrigine, GTC, IGE, generalized seizures, treatment

\section{Introduction}

Seizures in idiopathic generalized epilepsies are "conceptualized as originating at some point within, and rapidly engaging, bilaterally distributed networks. Such bilateral networks can include cortical and subcortical structures, but do not necessarily include the entire cortex". ${ }^{1-3}$

Patients with idiopathic generalized epilepsies may experience one or more seizure types, ie, primary generalized tonic-clonic seizures, absence seizures, and myoclonic seizures. ${ }^{4}$ Primary generalized tonic-clonic seizures are the most common generalized seizure type and the one that is the easiest to monitor clinically. In the initial, tonic phase, patients experience muscle contraction and body stiffening, which is followed by a clonic phase of rhythmic jerking of the face and limbs. Although primary generalized tonic-clonic seizures are the most visually recognizable seizures, other types exist and must be considered in the selection of appropriate treatments. Absence seizures usually manifest as brief episodes of altered consciousness, often with staring; they may occur multiple times per hour and are associated with the classic electroencephalographic (EEG) finding of generalized 3-4 Hz spike and wave discharges. Finally, patients with idiopathic generalized epilepsies frequently experience myoclonic seizures
Correspondence: Jerzy P Szaflarski Department of Neurology, University of Cincinnati Academic Health Center, 260 Stetson Street, Cincinnati,

$\mathrm{OH}$ 45267-0525, USA

$\mathrm{Tel}+\mathrm{I} 5 \mathrm{I} 35585440$

Fax + I $5 \mid 35584305$

Email Jerzy.Szaflarski@uc.edu 
or myoclonus that involves jerky movements of the body, sometimes affecting only one location, such as a single limb. Myoclonic seizures occur usually in patients with juvenile myoclonic epilepsy.

A recently published expert consensus statement suggested valproate, lamotrigine, and topiramate as the initial monotherapy choices for patients with primary generalized tonic-clonic seizures. ${ }^{5}$ Since then, double-blind studies have further documented the efficacy of levetiracetam in the treatment of idiopathic generalized epilepsy. ${ }^{6,7}$ Other idiopathic generalized epilepsy treatments may include zonisamide or vagus nerve stimulation. ${ }^{5,8-10}$ Overall, these treatments afford seizure freedom to approximately $80 \%$ of patients with idiopathic generalized epilepsies. ${ }^{11}$ Although lamotrigine is used for the treatment of all seizure types in patients with idiopathic generalized epilepsies, this review will focus on the use of lamotrigine in the treatment of primary generalized tonic-clonic seizures rather than myoclonic or absence seizures.

The mechanism of action of lamotrigine is not entirely elucidated. Studies have shown that it has multiple mechanisms of action, including blockage of voltage-gated $\mathrm{Na}^{+}$channels in the presynaptic neuronal membrane, stabilizing membranes, and inhibiting neurotransmitter release, principally glutamate. ${ }^{12}$ Lamotrigine is also thought to act on the presynaptic membrane via $\mathrm{N}$-type $\mathrm{Ca}^{+2}$ channel inhibition. ${ }^{13}$ Furthermore, studies have suggested that lamotrigine exerts an antiepileptic effect by acting upon the postsynaptic neuronal membrane via suppression of postsynaptic $\alpha$-amino-3-hydroxy-5-methyl-4-isoxazole propionic acid (AMPA) receptors. ${ }^{14}$ Although studies provide evidence for various drug targets, lamotrigine is one of many medications that are utilized for their clinical efficacy without a fully elucidated mechanism of action.

The average elimination half-life of immediate-release lamotrigine (LTG-IR) is approximately 24 hours when used in monotherapy. However, there is a sizeable variation in metabolism among individual patients that may produce large interindividual variations in levels. ${ }^{15}$ Furthermore, the elimination half-life of lamotrigine varies depending on coadministered medications, and the interactions with enzyme-inducing antiepileptic drugs or valproate are the most widely recognized. To attenuate such variations in serum concentration and to reduce the risk of both subtherapeutic and toxic serum levels, an enteric-coated extended-release formulation of lamotrigine (LTG-XR) was developed. LTG-XR has a modified-release eroding matrix to control its dissolution rate, ${ }^{16}$ which leads to changes in absorption rates (time to peak plasma concentration $\left[\mathrm{T}_{\max }\right] 1-1.5$ hours for LTG-IR versus 4-11 hours for LTG-XR). ${ }^{17}$ Despite the changes in $\mathrm{T}_{\max }$, the bioavailability of LTG-XR and LTG-IR is similar, except for patients taking enzyme-inducing antiepileptic drugs in whom the bioavailability of LTG-XR is $21 \%$ lower; the clinical importance of this finding is not clear. ${ }^{17}$ Its levels have been shown to be unaffected by high-fat meals, suggesting that the LTG-XR form is not significantly lipophilic. ${ }^{18}$ Studies have shown that lamotrigine is eliminated via hepatic $\mathrm{N}_{2}$-glucuronidation. ${ }^{19}$

LTG-XR may be preferable to LTG-IR for several reasons. Most obviously, the less frequent dosing of LTG-XR may enhance patient compliance and therefore provide better seizure control. For example, it is known that compliance decreases from $79 \%$ to $69 \%$ with once-daily versus twicedaily medication dosing. ${ }^{20}$ In one study, $71 \%$ of epilepsy patients reported missing at least one dose of medication, which precipitated seizure(s) in $45 \%$ of cases. ${ }^{21}$ Furthermore, based on a pharmacokinetic model, delayed dosing of LTG-XR (as in, eg, missing the dose by up to 12 hours in a patient taking valproic acid or up to five hours in a patient taking enzyme-inducing antiepileptic drugs) has been predicted to yield clinically manageable fluctuations in serum concentration with a less than $20 \%$ drop in peak plasma concentration. ${ }^{22}$ As compared with LTG-IR, LTG-XR has been demonstrated to reduce fluctuations in daily trough-to-peak serum concentrations. ${ }^{17}$ Both formulations have similar side effect profiles, with the most adverse events being serious skin rashes and hypersensitivity reactions. Suicidal ideation, acute organ failure, and blood dyscrasias have been reported with both formulations as serious adverse effects. Less severe, but more frequently observed, side effects for both formulations of lamotrigine include dizziness, tremor, vomiting, and diplopia. ${ }^{17,23}$ Headache, ataxia, blurred vision, somnolence, and rhinitis have also been reported with the LTG-IR formulation. Finally, both formulations carry the same drug interactions, including increased blood concentrations with the simultaneous use of valproic acid and decreased blood concentrations with concomitant use of carbamazepine, phenytoin, phenobarbital, primidone, rifampin, or oral estrogen-containing contraceptives.

Because the LTG-XR formulation may be preferable in some cases as indicated above (eg, to improve compliance or to decrease the probability of seizures resulting from inadvertently missed doses), guidelines for successful conversion from LTG-IR to LTG-XR have been proposed. Studies have demonstrated that the conversion process is quite logical and minimally complicated. Maintenance of steady-state and 
trough concentrations can be accomplished with equivalent daily milligrams of either formulation. ${ }^{17}$

LTG-IR was initially approved for the adjunctive treatment of partial seizures in 1994. Four years later, it was approved as adjunctive therapy for Lennox-Gastaut syndrome and as monotherapy for the treatment of partial seizures. Further expanding its utility, it was approved for the adjunctive treatment of primary generalized tonic-clonic seizures in 2006. LTG-XR was approved for adjunctive treatment of partial seizures in 2009 and for the adjunctive treatment of primary generalized tonic-clonic seizures in 2010. Although both LTG-IR and LTG-XR are approved for use as adjunctive treatment of partial seizures and primary generalized tonicclonic seizures, LTG-IR has been approved for a wider age range, starting with patients at the age of two years, whereas LTG-XR is only approved for those over 13 years of age. Finally, LTG-IR is approved for monotherapy in primary generalized tonic-clonic seizures, whereas LTG-XR does not have such an approval.

\section{Lamotrigine in clinical studies Randomized controlled trials}

Several randomized controlled studies of lamotrigine efficacy have been conducted. This review presents the data regarding the efficacy of lamotrigine in primary generalized tonicclonic seizures, with a special focus on the use of LTG-XR. Therefore, we will first review the studies that have led to LTG-IR/-XR approval for the treatment of primary generalized tonic-clonic seizures followed by other studies.

\section{Immediate-release lamotrigine}

One of the first reports on the use of LTG-IR in idiopathic generalized epilepsy examined its efficacy in patients with treatment-resistant seizures using a double-blind, placebocontrolled, crossover design. Overall, $50 \%$ of the patients had at least $50 \%$ reduction in primary generalized tonic-clonic seizures and $33 \%$ had $50 \%$ or more reduction in absence seizures when compared with placebo. Use of LTG-IR led to a statistically significant decrease in primary generalized tonic-clonic $(P=0.003)$ and absence seizures $(P<0.001)$, with 23/26 patients later continuing in an open-label study. ${ }^{24}$ In a regulatory, double-blinded study, 117 patients aged 2-55 years with idiopathic generalized epilepsies and experiencing medication-refractory primary generalized tonic-clonic seizures were randomized to receive LTG-IR or placebo. In the combined escalation and maintenance phases, the median percent reduction in generalized seizures was $66.5 \%$ with LTG-IR versus $34.2 \%$ with placebo $(P=0.006)$.
These numbers were slightly lower in the escalation phase (60.6\% versus $32.8 \% ; P=0.038)$ and higher in the maintenance phase $(81.9 \%$ versus $43.0 \% ; P=0.006) .{ }^{25}$ Furthermore, approximately $72 \%$ of LTG-IR patients were considered responders compared with $49 \%$ of patients receiving placebo $(P=0.014)$. A similar pattern of results was observed for all generalized seizures. Post hoc analysis of these data revealed that LTG-IR was superior to placebo in median days to third, sixth, ninth, and 12 th seizure $(P<0.022$ for all $),{ }^{26}$ with its efficacy evident within the first two weeks of therapy initiation (when compared with placebo, $P=0.036$ ). ${ }^{27}$ The data from the original trial were later reanalyzed with a focus on the pediatric population. ${ }^{28}$ This analysis included 45 children and adolescents, aged 2-19 years, who were randomly assigned to receive either LTG-IR or placebo. A significant decrease in median generalized seizure frequency between the groups was noted ( $77 \%$ versus $40 \% ; P=0.044)$, with median primary generalized tonic-clonic seizure counts per month of 0.4 versus 2.5 during the entire treatment period $(P=0.007)$, and higher treatment success in the escalation phase $(0.7$ versus $3.6 ; P=0.008)$ than in the maintenance phase $(0.3$ versus $2.0 ; P=0.005)$. Based on these studies, LTG-IR was deemed efficacious for the treatment of primary generalized tonic-clonic seizures in children and adults.

\section{Extended-release lamotrigine}

Although there is evidence for the efficacy of LTG-IR for the treatment of primary generalized tonic-clonic seizures, the arrival of the LTG-XR formulation necessitated new efficacy and tolerability studies. In a randomized, doubleblind trial of LTG-XR in patients with primary generalized tonic-clonic seizures, a pragmatic intent-to-treat analysis was used to compare the efficacy of LTG-XR $(n=70)$ with placebo $(\mathrm{n}=73)$. The seizure-free rate was $44 \%$ versus $12 \%$ $(P<0.0001)$, and the responder rate for $50 \%$ reduction in seizure frequency during the maintenance phase was $70 \%$ versus $38 \%(P=0.0002) .{ }^{29}$ Another randomized, doubleblind, placebo-controlled, parallel-group trial assessed the efficacy of LTG-XR in lamotrigine-naïve patients aged 13 years and over with primary generalized tonic-clonic seizures. The study compared 153 patients who were randomized to LTG-XR $(n=76)$ with placebo $(n=77)$. Although the primary generalized tonic-clonic seizure frequencies per week at baseline were similar between the LTG-XR (0.8) and placebo (0.6) groups, the median percent decrease from baseline during the escalation phase was $62 \%$ for LTG-XR versus $26 \%$ for placebo $(P=0.0003)$, and $89 \%$ for LTG-XR versus $33 \%$ for placebo $(P<0.0001)$ in the maintenance 
phase. The decrease in seizure frequency related to LTG-XR administration was $76 \%$ versus $30 \%$ in the placebo group $(P<0.0001)$. Notably, this study also addressed the rate of seizure freedom with extended-release adjunctive therapy. The rate of seizure freedom in the escalation phase was $23 \%$ and $11 \%$ in the respective groups $(P=0.075)$, and during the maintenance phase the rate of seizure freedom was $46.4 \%$ and $14.3 \%$ for the extended-release and placebo groups $(P=0.034)$, respectively. ${ }^{30}$

\section{Other immediate-release lamotrigine studies}

LTG-IR appears to be of comparable efficacy with another first-line antiepileptic drug, valproate, when treating newly diagnosed individuals with generalized epilepsies. ${ }^{31}$ In the open-label LAM-SAFE study, patients with new-onset epilepsy were stratified to either a carbamazepine-lamotrigine arm (patients with focal onset seizures) or to a valproatelamotrigine arm (patients with idiopathic generalized epilepsy). In the idiopathic generalized epilepsy (second) arm, 63 patients received either valproate $(n=30)$ or lamotrigine $(n=33)$. During the 24 weeks of study participation, $61 \%$ of the lamotrigine patients and $84 \%$ of the valproate patients became seizure-free (difference not significant). ${ }^{32}$ Another controlled trial in patients with generalized onset or unclassified seizures compared the efficacy of valproate, LTG-IR, and topiramate. ${ }^{33}$ In this study, patients were randomly assigned to valproate, LTG-IR, or topiramate and followed up for several years after therapy initiation. In all enrolled patients, time to treatment failure for valproate was greater than for topiramate, ie, hazard ratio (HR) $1.57,95 \%$ confidence interval (CI) 1.19-2.08, but there was no difference between valproate and LTG-IR (HR 1.25, 95\% CI 0.94-1.68). When the same analysis was conducted in patients with definite idiopathic generalized epilepsy, valproate was significantly better than both LTG-IR (HR 1.55, 95\% CI 1.07-2.24) and topiramate (HR 1.89, 95\% CI 1.32-2.70). Furthermore, for time to 12-month remission, valproate was significantly better than LTG-IR overall (HR 0.76, 95\% CI 0.62-0.94) and for the idiopathic generalized epilepsy patients (HR 0.68, 95\% CI $0.53-0.89)$. There was no difference between valproate and topiramate in either the analysis overall or for the subgroup with idiopathic generalized epilepsy.

A prospective, open-label, video-EEG study examined the efficacy of LTG-IR as an add-on or monotherapy in idiopathic generalized epilepsy. ${ }^{34}$ Of the 47 patients enrolled, 12 had childhood absence epilepsy (nine became seizure-free), 12 had juvenile absence epilepsy (10 became seizure-free), and 15 had juvenile myoclonic epilepsy (seven became seizure-free). The remaining patients had various other idiopathic generalized epilepsy syndromes, including grand mal on awakening; seizure-free status was less frequent in these patients.

In a retrospective study, the efficacy of LTG-IR was compared with the efficacy of valproate in patients with idiopathic generalized epilepsy. ${ }^{35}$ More patients remained on valproate monotherapy at one and two years $(89 \%$ and $83 \%$, respectively) than on lamotrigine monotherapy $(69 \%$ and $57 \%$ ). Although valproate showed comparable efficacy in all idiopathic generalized epilepsy syndromes, lamotrigine appeared to be more efficacious in patients with childhood and juvenile absence epilepsies than in juvenile myoclonic epilepsy. ${ }^{35}$ This is consistent with previous reports of lamotrigine potentially aggravating myoclonic epilepsies. ${ }^{36,37}$

Because valproic acid has traditionally been a first-line antiepileptic drug in the treatment of juvenile myoclonic epilepsy, several studies have compared LTG-IR with valproate for the treatment of this disorder. In one open-label study, patients aged $\geq 12$ years with newly diagnosed juvenile myoclonic epilepsy (drug-naïve or on an incorrect antiepileptic drug due to misdiagnosis) were enrolled. ${ }^{38}$ LTG-IR was titrated up to a maximum dosage of 100-500 mg/day, followed by a 24-week treatment phase with LTG-IR adjustments as needed. Of the 29 analyzable patients, 58\% experienced a reduction from baseline seizure frequency of at least $50 \%$ in days with myoclonus, and $56 \%$ and $38 \%$ of patients experienced a reduction of at least $50 \%$ in the frequency of primary generalized tonic-clonic and absence seizures, respectively. LTG-IR was also used in an open-label study of juvenile myoclonic epilepsy patients who had failed treatment with valproate. ${ }^{39}$ Investigators found that $50 \%$ of the 63 enrolled patients had a reduction in adverse events as a result of the change in anticonvulsants, and $67 \%$ had improvement in global clinical status; $76 \%$ of patients rated LTG-IR better than valproate. Another study retrospectively evaluated the efficacy of LTG-IR in monotherapy for a variety of pediatric epilepsies, including idiopathic generalized epilepsy. Of the 83 children enrolled, 32 had idiopathic generalized epilepsy, and $36 \%$ of them became seizure-free on LTG-IR, including all children with the diagnosis of juvenile myoclonic epilepsy. ${ }^{40}$ Buchanan evaluated 12 patients with juvenile myoclonic epilepsy who had either failed valproate or refused to take it; five patients became seizure-free after LTG-IR was introduced. ${ }^{41}$ In another study, absence seizures were completely eliminated in refractory cases while using LTG-IR in combination with one other anticonvulsant. ${ }^{42}$ In the same 
study, all of the patients with juvenile myoclonic epilepsy experienced complete seizure freedom when using LTG-IR in combination therapy. In a pilot study, Timmings and Richens evaluated the efficacy of LTG-IR as second-line monotherapy in 17 patients with juvenile myoclonic epilepsy. ${ }^{43}$ They showed that there were no differences in seizure control following a four-week, single-blind, placebo add-on period, nor were there differences in a later randomization to continued therapy with valproate or a switch to LTG-IR in a doubledummy, double-blind, 12-week study.

In a longitudinal study of 10 years, lamotrigine was administered to patients with medication-resistant idiopathic generalized epilepsy. ${ }^{44}$ The authors observed that $33 \%$ of patients became seizure-free, and $43.6 \%$ had improvement in seizure control; $23 \%$ dropped out of the study due to insufficient seizure control, and $17.9 \%$ experienced adverse effects that necessitated medication cessation. Meo et al reported a similar magnitude of seizure reduction rates with better tolerability. ${ }^{45}$

Although LTG-XR is approved by the US Food and Drug Administration as adjunctive therapy for both partial and primary generalized tonic-clonic seizures, its indication for myoclonic and absence seizures is not yet in place. However, if the success with LTG-IR in the treatment of idiopathic generalized epilepsy may be extrapolated to LTG-XR, studies showing its efficacy in the treatment of myoclonic and absence seizures are likely to be imminent.

\section{Conclusions}

LTG-IR and LTG-XR have provided a novel treatment option for patients with epilepsy, a disease that has multiple comorbidities and is potentially threatening to quality of life. ${ }^{46}$ A medication such as lamotrigine that is useful for application in multiple forms of epilepsy is especially advantageous. The efficacy that has been attained in clinical studies, as well as its superior tolerability profile, makes lamotrigine an important antiepileptic drug choice. More research is required to elucidate all possible indications for LTG-XR use. Some controversy still exists regarding its efficacy, particularly in the treatment of myoclonic seizures in juvenile myoclonic epilepsy, and therefore further studies are needed.

In the published studies, lamotrigine has rarely produced severe adverse reactions, although the risk of life-threatening rash must not be ignored. Generally, this is a well tolerated antiepileptic drug, with the most common side effects including headache, nausea, and dizziness. These are, at least in part, dose-related, and may be endurable in many cases. Lamotrigine is also easily managed, with relatively few drug interactions, as compared with some of the older antiepileptic drugs.

The straightforward conversion between LTG-IR and LTG-XR dosing is also advantageous. A more manageable dosing regimen, enhancing patient compliance and with steadier serum concentrations, makes LTG-XR an attractive option. The LTG-XR formulation appears to have a similar degree of efficacy to and comparable tolerability profile with that of LTG-IR. Further research is still necessary in order to generate approval by the Food and Drug Administration for all potential applications of LTG-XR. However, these advancements are generating hope in those affected by epilepsy.

\section{Disclosure}

Dr Steinbaugh does not report any conflicts of interests. Dr Szaflarski has served as a paid speaker for Glaxo Smith Kline.

\section{References}

1. Bai X, Vestal M, Berman R, et al. Dynamic time course of typical childhood absence seizures: EEG, behavior, and functional magnetic resonance imaging. J Neurosci. 2010;30:5884-5893.

2. Berg AT, Berkovic SF, Brodie MJ, et al. Revised terminology and concepts for organization of seizures and epilepsies: Report of the ILAE Commission on Classification and Terminology, 2005-2009. Epilepsia. 2010;51:676-685.

3. Szaflarski JP, DiFrancesco M, Hirschauer T, et al. Cortical and subcortical contributions to absence seizure onset examined with EEG/fMRI. Epilepsy Behav. 2010;18:404-413.

4. Engel J Jr. Report of the ILAE classification core group. Epilepsia. 2006;47:1558-1568.

5. Karceski S, Morrell MJ, Carpenter D. Treatment of epilepsy in adults: Expert opinion. Epilepsy Behav. 2005;7 Supp1 1:S1-S64.

6. Berkovic SF, Knowlton RC, Leroy RF, Schiemann J, Falter U. Placebocontrolled study of levetiracetam in idiopathic generalized epilepsy. Neurology. 2007;69:1751-1760.

7. Noachtar S, Andermann E, Meyvisch P, Andermann F, Gough WB, Schiemann-Delgado J. Levetiracetam for the treatment of idiopathic generalized epilepsy with myoclonic seizures. Neurology. 2008;70: 607-616.

8. Bergey GK. Evidence-based treatment of idiopathic generalized epilepsies with new antiepileptic drugs. Epilepsia. 2005;46 Suppl 9: 161-168.

9. $\mathrm{Ng} \mathrm{M}$, Devinsky O. Vagus nerve stimulation for refractory idiopathic generalised epilepsy. Seizure. 2004;13:176-178.

10. Szaflarski JP. Effects of zonisamide on the electroencephalogram of a patient with juvenile myoclonic epilepsy. Epilepsy Behav. 2004;5:1024-1026.

11. Szaflarski JP, Lindsell CJ, Zakaria T, Banks C, Privitera MD. Seizure control in patients with idiopathic generalized epilepsies: EEG determinants of medication response. Epilepsy Behav. 2010;17: 525-530.

12. Leach MJ, Marden CM, Miller AA. Pharmacological studies on lamotrigine, a novel potential antiepileptic drug: II. Neurochemical studies on the mechanism of action. Epilepsia. 1986;27:490-497.

13. Wang SJ, Huang CC, Hsu KS, Tsai JJ, Gean PW. Presynaptic inhibition of excitatory neurotransmission by lamotrigine in the rat amygdalar neurons. Synapse. 1996;24:248-255.

14. Lee CY, Fu WM, Chen CC, Su MJ, Liou HH. Lamotrigine inhibits postsynaptic AMPA receptor and glutamate release in the dentate gyrus. Epilepsia. 2008;49:888-897. 
15. Werz MA. Pharmacotherapeutics of epilepsy: Use of lamotrigine and expectations for lamotrigine extended release. Ther Clin Risk Manag. 2008;4:1035-1046.

16. Rheims S, Ryvlin P. Once-daily lamotrigine extended release for epilepsy management. Expert Rev Neurother. 2009;9:167-173.

17. Tompson DJ, Ali I, Oliver-Willwong R, et al. Steady-state pharmacokinetics of lamotrigine when converting from a twice-daily immediaterelease to a once-daily extended- release formulation in subjects with epilepsy (The COMPASS Study). Epilepsia. 2008;49:410-417.

18. Kustra R, Tompson D, Job S, van Landingham K. A summary of the pharmacokinetic characteristics of lamotrigine extended-release tablets. Epilepsia. 2007;48:371.

19. Rowland A, Elliot DJ, Williams JA, Mackenzie PI, Dickinson RG, Miners JO. In vitro characterization of lamotrigine N2-glucuronidation and the lamotrigine-valproic acid interaction. Drug Metab Dispos. 2006;34:1055-1062.

20. Claxton AJ, Cramer J, Pierce C. A systematic review of the associations between dose regimens and medication compliance. Clin Ther. 2001;23:1296-1310.

21. Cramer JA, Glassman M, Rienzi V. The relationship between poor medication compliance and seizures. Epilepsy Behav. 2002;3:338-342.

22. Kustra RP, Tompson D, Wright J, Messenheimer J. Modeling and simulation of the phamacokinetics of once daily lamotrigine extended release to support the evaluation of dosing scenarios and comparisons with twice daily immediate-release lamotrigine. Epilepsia. 2008;49:439.

23. Vuong A, Hammer AE, Kerls SP, Caldwell PT, Messenheimer JA. Long term safety data with lamotrigine extended-release: A meta-analysis of 3 controlled clinical trials. Epilepsia. 2009;50:413.

24. Beran RG, Berkovic SF, Dunagan FM, et al. Double-blind, placebocontrolled, crossover study of lamotrigine in treatment-resistant generalised epilepsy. Epilepsia. 1998;39:1329-1333.

25. Biton V, Sackellares JC, Vuong A, Hammer AE, Barrett PS, Messenheimer JA. Double-blind, placebo-controlled study of lamotrigine in primary generalized tonic-clonic seizures. Neurology. 2005;65:1737-1743.

26. French J, Temkin N, Hammer A, van Landingham K. Time to the nth seizure analysis of lamotrigine as adjunctive therapy in subjects with primary generalized epilepsy. Epilepsia. 2007;48 Suppl 6:77-78.

27. Biton V, Vuong A, Kerls SP, Hammer AE, Messenheimer J. Onset of efficacy with lamotrigine in primary generalized tonic-clonic seizures (PGTC): A randomized, placebo controlled study. Epilepsia. 2005; 46 Suppl 8:206.

28. Trevathan E, Kerls SP, Hammer AE, Vuong A, Messenheimer JA. Lamotrigine adjunctive therapy among children and adolescents with primary generalized tonic-clonic seizures. Pediatrics. 2006;118: e371-e378.

29. Hammer A, Vuong A, Kerls S, Caldwell P, Messenheimer J. Pragmatic intent-to-treat analysis for lamotrigine extended-release from 3 epilepsy clinical trials. Epilepsia. 2009;413-414.

30. Biton V, Vuong A, Hammer A, Adams BE, Kerls S, Messenheimer J. Preliminary results with once-daily lamotrigine extended-release as adjunctive therapy in patients with primary generalized tonic-clonic seizures. In: 60th Annual Meeting of the American Academy of Neurology, Chicago, IL, 2008 Apr 12-19.
31. Steinhoff BJ, Ueberall MA, Siemes H, et al; The LAM-SAFE Study: Lamotrigine versus carbamazepine or valproic acid in newly diagnosed focal and generalised epilepsies in adolescents and adults. Seizure. 2005; 14:597-605.

32. Steinhoff BJ, Ueberall MA, Siemes H, Kurlemann G, Schmitz B, Bergmann L. The LAM-SAFE Study: Lamotrigine versus carbamazepine or valproic acid in newly diagnosed focal and generalised epilepsies in adolescents and adults. Seizure. 2005;14:597-605.

33. Marson AG, Al-Kharusi AM, Alwaidh M, et al. The SANAD study of effectiveness of valproate, lamotrigine, or topiramate for generalised and unclassifiable epilepsy: An unblinded randomised controlled trial. Lancet. 2007;369:1016-1026.

34. Gericke CA, Picard F, de Saint-Martin A, Strumia S, Marescaux C, Hirsch E. Efficacy of lamotrigine in idiopathic generalized epilepsy syndromes: A video-EEG-controlled, open study. Epileptic Disord. 1999;1:159-165.

35. Mazurkiewicz-Beldzinska M, Szmuda M, Matheisel A. Longterm efficacy of valproate versus lamotrigine in treatment of idiopathic generalized epilepsies in children and adolescents. Seizure. 2010;19:195-197.

36. Crespel A, Genton P, Berramdane M, et al. Lamotrigine associated with exacerbation or de novo myoclonus in idiopathic generalized epilepsies. Neurology. 2005;65:762-764.

37. Maiga Y, Nogues B, Guillon B. [Exacerbation of tonicoclonic seizures in a juvenile myoclonic epileptic taking lamotrigine]. [in French] Rev Neurol (Paris). 2006;162:1125-1127.

38. Schimschock JR, Hammer AE, Kustra RP, Messenheimer JA. Effects of lamotrigine monotherapy in patients with newly diagnosed juvenile myoclonic epilepsy: An open-label study. Curr Ther Res Clin Exp 2005;66:230-237.

39. Morris GL, Hammer AE, Kustra RP, Messenheimer JA. Lamotrigine for patients with juvenile myoclonic epilepsy following prior treatment with valproate: Results of an open-label study. Epilepsy Behav. 2004;5:509-512.

40. Barron TF, Hunt SL, Hoban TF, Price ML. Lamotrigine monotherapy in children. Pediatr Neurol. 2000;23:160-163.

41. Buchanan N. The use of lamotrigine in juvenile myoclonic epilepsy. Seizure. 1996;5:149-151.

42. Fallah R, Islami Z, Tavakoli N. Efficacy and safety of lamotrigine in refractory epilepsy of children. East J Med. 2008;13:7-12.

43. Timmings P, Richens A. Efficacy of lamotrigine as monotherapy for juvenile myoclonic epilepsy: Pilot study results. Epilepsia. 1993;34:160.

44. Garcia-Escriva A, Lopez-Hernandez N, Alvarez-Sauco M, et al. [Lamotrigine in refractory epilepsy]. Rev Neurol. 2004;38:301-303. Spanish.

45. Meo R, Bilo L, Mariniello A, et al. Lamotrigine as add-on therapy in drug resistant generalized epilepsies: Efficacy and tolerability. Lega Italiana contro l'Epilessia. 1999;106-107:309-311.

46. Szaflarski JP, Szaflarski M. Seizure disorders, depression, and healthrelated quality of life. Epilepsy Behav. 2004;5:50-57.
Drug Design, Development and Therapy

\section{Publish your work in this journal}

Drug Design, Development and Therapy is an international, peerreviewed open-access journal that spans the spectrum of drug design and development through to clinical applications. Clinical outcomes, patient safety, and programs for the development and effective, safe, and sustained use of medicines are a feature of the journal, which

\section{Dovepress}

has also been accepted for indexing on PubMed Central. The manuscript management system is completely online and includes a very quick and fair peer-review system, which is all easy to use. Visit http://www.dovepress.com/testimonials.php to read real quotes from published authors. 